2016 - Volume: 17 Number: 3

Page: 551 - 562

DOI: $10.18038 /$ btda. 63537

Received: 30 March 2016 Revised: 26 July 2016 Accepted: 05 August 2016

\title{
SELECTIVE AND SENSITIVE ELECTROCHEMICAL SENSING OF SEROTONIN IN HUMAN BLOOD SERUM BY MEANS OF ELECTROCHEMICALLY TREATED PENCIL GRAPHITE ELECTRODE
}

\author{
Ali ÖZCAN* \\ Department of Chemistry, Faculty of Science, Anadolu University, 26470 Eskişehir, Turkey
}

\begin{abstract}
Serotonin (SER) is an important monoamino-neurotransmitter found in body fluids. The determination of SER is a very important issue because its concentration behaves as an indicator in the diagnosis of many illnesses. Moreover, its concentration level in body fluids is very low. Therefore, the development of new sensitive and selective analytical methods is a very hot topic. In this study, voltammetric determination of SER in blood serum was investigated by electrochemically treated pencil graphite (ETPG) electrode for the first time. The electro-catalytic effect of ETPG decreased the oxidation potential of SER from $+0.52 \mathrm{~V}$ to $+0.48 \mathrm{~V}$. The high surface area of ETPG together with its electro-catalytic effect increased the oxidation peak current values tremendously (forty times) according to bare PG. Moreover, the ETPG electrode allowed the oxidation of SER and uric acid (UA) at separate potential values that was not possible in the case of bare PG. It was observed that the interference effect of UA, which was a major component of blood serum, was negligible at $\mathrm{pH}$ value of 1.6. The ETPG showed good performance for the determination of SER with a linear range between $0.005 \mu \mathrm{M}$ and $1.0 \mu \mathrm{M}$. The relative standard deviation of ten repetitive measurements of $1.0 \mu \mathrm{M}$ SER was calculated as $2.8 \%$ indicating highly reproducible response of ETPG electrode. The detection limit was calculated as $1.0 \mathrm{nM}(\mathrm{S} / \mathrm{N}=3)$. The performance of the developed voltammetric method was successfully tested in real samples (blood serum) and it was compared with a previously published HPLC method.
\end{abstract}

Keywords: Serotonin, Electrochemically treated pencil graphite, Uric acid, Blood serum, Voltammetry

\section{INTRODUCTION}

Serotonin (SER), which is also called as 5-hydroxytryptamine, is a monoamine neurotransmitter. It has many important duties in central and peripheral nervous systems [1]. It controls and regulates many physiological functions like sexual activity, thermoregulation and sleep. Moreover, it plays a prominent role in some psychopathological states such as drug dependency, depression, alcoholism and anxiety [2]. Many gastrointestinal disorders such as food hypersensitivity, irritable bowel syndrome and inflammatory bowel disease were also related with the SER levels in body fluids [3]. It was also reported that abnormal concentrations of SER in plasma had negative effects on the central nervous system [4]. Therefore, determination of SER level in body fluids is a very important issue for the diagnosis and monitoring of many illnesses related with SER. While the chromatographic and spectroscopic methods are very effective in the determination of SER, they require high cost instruments, sample pretreatment and long analysis durations, which restrict their everyday usage.

The electroactivity of SER has encouraged recently the researchers to develop voltammetric determination strategies, which are mainly based on the electrocatalytic oxidation of SER on different electrode materials. Most of these studies focused on the modification of glassy carbon (GC) electrodes. In this manner, carbon nanotube film [2], C-undecylcalix[4]resorcinarene [5], electrochemically reduced graphite oxide-porphyrin [6], carbon nanofibers [7] and graphene nanomaterials [8] were investigated as a modifier for GC. In addition, $\mathrm{Co}(\mathrm{OH})_{2}$ nanoparticles-multiwalled carbon nanotube modified carbon ionic liquid electrode [9], and polymelamine modified edge plane pyrolytic graphite [10] electrodes were also investigated. The regeneration of the electrode surface is a very important issue in the subsequent analysis since the oxidized molecules can form different monomeric or polymeric intermediates which may change the surface characteristics of the electrodes.

*Corresponding Author: aozcan3@anadolu.edu.tr 
The disposable electrodes may be an alternative for the modified and unmodified GC electrodes to overcome the regeneration drawbacks. Pencil leads can be regarded as alternative electrode materials for the electroanalytical applications because of their promising properties such as their commercial availability, low cost and high reproducibility. The surface characteristics of the electrodes, which mainly affect the electrode sensitivity and selectivity, can be changed by various ways. One of the possible ways of changing surface characteristics of bare electrodes is the electrochemical treatment [11]. The electrochemical treatment of PG electrodes at different conditions forms the electrochemically treated pencil graphite electrode (ETPG), which has different surface properties. The analytical application of ETPG was investigated in our previous studies focusing on the sensitive and selective detection of dopamine [12], uric acid (UA) [13], paracetamol [14] and tryptophan [15] in blood serum. These literatures indicated that the ETPG electrodes had different surface functionalities, which increased the adsorption of analytes on the electrode surface and showed catalytic effect on the electrooxidation of analytes. The main functional groups on the ETPG electrode carboxyl, carbonyl and hydroxyl groups. The carbonyl and carboxyl groups show electro-catalytic activity and the carboxyl group behaves as a negatively charged barrier. Because of these properties, the ETPG electrode is a promising candidate to detect the low levels of SER in blood serum samples. Especially the carboxyl group of the ETPG is a possible attractive site for the amino group of SER. Here, the determination of SER has been examined by ETPG electrode for the first time in the literature. The oxidation peak currents of SER increased forty times in the case of ETPG, indicating an electrocatalytical effect. On the contrary to bare PG electrode, the ETPG successfully discriminated the oxidation peak potentials of SER and UA. A very low detection limit value $(0.001 \mu \mathrm{M})$ for SER was achieved. The detection of SER was successfully achieved in blood serum samples containing high concentrations of UA, which is an interfering molecule in the case of bare PG electrode.

\section{EXPERIMENTAL SECTION}

\subsection{Chemicals}

Serotonin (5-Hydroxytryptamine creatinine sulfate monohydrate) and uric acid (99\%) were purchased from Aldrich. All chemicals used in the experiments were used as received. Ultra pure deionized water was used in the preparation of standard solutions. The standard solutions of SER were prepared freshly each day using a minimum amount of $1.0 \mathrm{M} \mathrm{HCl}$ and stored at $+4{ }^{\circ} \mathrm{C}$.

The real samples (blood serum) were taken from local hospitals. They were analyzed both by the proposed method and a previously published HPLC method. Blood serum samples were directly used in the voltammetric analysis without any pretreatment. On the other hand, in the case of HPLC analysis, protein-free blood serum samples were prepared by precipitating the serum proteins. For this purpose, blood serum samples were mixed in an appropriate amount of $30 \% \mathrm{HClO}_{4}$ solution. After that, the samples were vortexed for $10 \mathrm{~s}$ and centrifuged at $12000 \mathrm{xg}$ for $10 \mathrm{~min}$. Then, $20 \mu \mathrm{L}$ of the supernatant was injected onto the column and HPLC analysis performed. To compare the results, the same proteinfree serum samples were also analysed with the proposed method. In adsorptive stripping differential pulse voltammetry (ASDPV) analysis, $1000 \mu \mathrm{L}$ of the supernatant was transferred into the electrochemical cell and it was diluted up to ten-folded with $0.1 \mathrm{M} \mathrm{H}_{3} \mathrm{PO}_{4}$ solution containing $0.02 \mathrm{M}$ $\mathrm{LiClO}_{4}$. The standard addition method was used in both ASDPV and HPLC analysis to evaluate the SER content of blood serum samples.

\subsection{Apparatus}

A conventional three-electrode cell was used in the electrochemical studies. A saturated calomel electrode (SCE) and a platinum electrode were served as a reference and an auxiliary electrode, respectively. The indicator electrode was a pencil graphite (PG) or an electrochemically treated pencil graphite (ETPG). ETPG electrodes were prepared by a Voltalab PST402 Potentiostat / Galvanostat. 
Voltammetric studies were conducted by an Autolab PGSTAT 100 Potentiostat / Galvanostat (EcoChemie, The Netherlands).

Tombo leads (diameter: $0.5 \mathrm{~mm}$ ) were obtained from local suppliers and used as PG electrodes. The obtained leads were cut into $3 \mathrm{~cm}$ long sticks and $1.2 \mathrm{~cm}$ was treated. A mechanical pencil was used as a holder.

\subsection{Electrochemical Treatment of PG Electrode}

The ETPG electrodes were prepared according to previously published procedure [12]. Briefly, the PG electrode was placed in an aqueous solution of $\mathrm{H}_{3} \mathrm{PO}_{4}(0.1 \mathrm{M})$ and potential sweeping was performed between $-0.3 \mathrm{~V}$ and $+2.0 \mathrm{~V}$ for 3 scans with a scan rate of $50 \mathrm{mV} \mathrm{s}^{-1}$. After that, the formed ETPG electrodes were washed with deionized water and stored in a vacuum desiccator until their use.

\subsection{Adsorptive Stripping Differential Pulse Voltammetry (ASDPV) Measurements}

The voltammetric determination of SER was performed by ASDPV using ETPG electrode. In order to accumulate SER on the electrode surface, the ETPG was incubated in a magnetically stirred solution of SER. After that, the electrode was hold for $2 \mathrm{~s}$ without stirring in the same solution. Finally, the adsorbed SER was stripped by the potential scanning from $+0.1 \mathrm{~V}$ to $+0.9 \mathrm{~V}$. Every measurement was repeated three times using a new electrode.

\subsection{High Performance Liquid Chromatography Analysis (HPLC)}

The SER concentration of the blood serum samples was also determined by HPLC analysis [16]. HPLC analysis was conducted by Agilent 1100 system equipped with an electrochemical detector. A reversed phase Inertsil ODS3 $(5 \mu \mathrm{m}, 4.6 \times 250 \mathrm{~mm})$ column, thermostated at $25{ }^{\circ} \mathrm{C}$, was used. Mobile phase contains a mixture of $0.1 \mathrm{M}$ sodium acetate buffer solution and methanol $(85: 15, \mathrm{v} / \mathrm{v})$. The $\mathrm{pH}$ of the mobile phase was adjusted to 4.2 by concentrated acetic acid. The flow rate of mobile phase is $0.8 \mathrm{~mL}$ $\mathrm{min}^{-1}$. The electrochemical detector was operated at a potential of $+0.6 \mathrm{~V}$ ( $\mathrm{vs} \mathrm{Ag} / \mathrm{AgCl}$ ).

\section{RESULTS AND DISCUSSION}

\subsection{Determination of Electrochemical Effective Surface Areas of PG and ETPG}

The electrochemical effective surface areas of PG and ETPG electrodes were determined by cyclic voltammetry using Randles-Sevcik equation (Eq. (1)).

$$
i_{p a}=2.69 \times 10^{5} n^{3 / 2} A C_{0} D_{R}^{1 / 2} v^{1 / 2}
$$

Here, $i_{p a}$ shows the anodic peak current. $n$ is the number of transferred electrons. A refers to the electrochemical effective surface area $\left(\mathrm{cm}^{2}\right) . D_{R}$ and $C_{0}$ show the diffusion coefficient and the concentration of redox probe, respectively. $v$ is the scan rate. According to this equation, a linear relationship can be obtained between the $i_{p a}$ and $v^{1 / 2}$ by performing cyclic voltammetry measurements of a redox probe. The slope of this line can be used in the determination of electrochemical effective surface area. The cyclic voltammetry measurements of $1.0 \mathrm{mM} \mathrm{Ru}\left(\mathrm{NH}_{3}\right)_{6} \mathrm{Cl}_{3}$ in $0.1 \mathrm{M} \mathrm{KCl}$ solution were performed at different potential scan rates to determine the electrochemical effective surface areas of PG and ETPG electrodes. The values of $D_{R}$ and $n$ for $1.0 \mathrm{mM} \mathrm{Ru}\left(\mathrm{NH}_{3}\right)_{6}{ }^{3+}$ in a $0.1 \mathrm{M} \mathrm{KCl}$ solution at $25{ }^{\circ} \mathrm{C}$ equal to $8.4 \times 10^{-6} \mathrm{~cm}^{2} \mathrm{~s}^{-1}$ [17] and 1, respectively. The slopes of the lines obtained from the relation between the $i_{p a}$ and $v^{1 / 2}$ for PG and ETPG electrodes were determined as $34.34 \times 10^{-6}$ and 134.35 x $10^{-6}$, respectively. Using these slopes and the given values for $\mathrm{Ru}\left(\mathrm{NH}_{3}\right)_{6} \mathrm{Cl}_{3}$, the electrochemical effective surface areas of PG and ETPG were calculated as $0.044 \mathrm{~cm}^{2}$ and $0.172 \mathrm{~cm}^{2}$, respectively. As 
can be seen, the electrochemical effective surface area of ETPG was almost 4 times higher than that of bare PG. This clearly verifies that the electrochemical treatment increases the effective surface area of PG electrode.

\subsection{Electrochemical Oxidation of SER}

The ASDPV was used as an electrochemical method throughout the study. This method consists of two steps, which are the adsorption of analyte on working electrode and stripping of the adsorbed analyte. Here, the ETPG and PG electrodes were kept in $0.1 \mathrm{M} \mathrm{H}_{3} \mathrm{PO}_{4}$ solution containing $0.02 \mathrm{M} \mathrm{LiClO}_{4}$ in the absence and presence of $5.0 \mu \mathrm{M}$ SER for $2 \mathrm{~min}$. After that, DPV measurements were performed at the same medium. The obtained results are shown in Figure 1. The corresponding voltammogram showed an oxidation peak at $+0.22 \mathrm{~V}$ (vs SCE) in the absence of SER in Figure 1A. This peak arises from the redox behavior of surface functional groups of the ETPG, which formed on the PG during the electrochemical treatment [11]. When 5.0 $\mu \mathrm{M}$ SER was added into the blank solution, two oxidation peaks were observed at the peak potential values of $+0.48 \mathrm{~V}$ and $+0.69 \mathrm{~V}$ (vs. SCE) in addition to the oxidation peak of ETPG in Figure 1B. It can be easily seen that these peaks may arise from the oxidation of SER on the ETPG. On the other hand, the oxidation of SER took place at $+0.52 \mathrm{~V}$ in the case of bare PG at the same conditions in Figure 1C. These results indicate that the ETPG electrode shows a catalytic effect on the electrochemical oxidation of SER by decreasing the oxidation potential almost $40 \mathrm{mV}$. It can also be seen that the oxidation peak current values of SER was increased almost forty times at ETPG electrode according to untreated PG. These results indicate that the current increase is mainly arisen from the electrocatalytical effect of ETPG towards SER because the electrochemical effective surface area of ETPG is only 4 times higher than that of PG electrode.

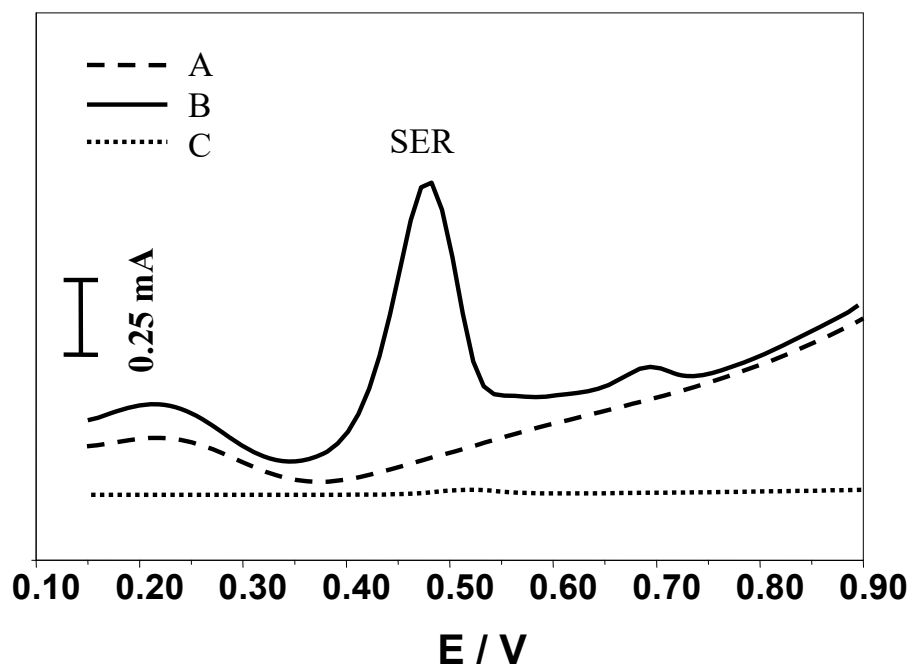

Figure 1. The ASDPVs of ETPG obtained in the mixture of $0.1 \mathrm{M} \mathrm{H}_{3} \mathrm{PO}_{4}$ and $0.02 \mathrm{M} \mathrm{LiClO}_{4}$ solution in the absence (A) and presence of 5.0 $\mu \mathrm{M}$ SER (B). C shows the DPV of bare PG obtained as $\mathrm{B}$. Before the measurements, the electrodes were immersed for 3 min into the solution at stirring conditions $(250 \mathrm{rpm})$

\subsection{The Effect of pH on the Electrochemical Oxidation of SER at ETPG Electrode}

It is well known that SER generally coexist with ascorbic acid (AA), dopamine (DA) and UA in biological fluids and these molecules are also electro-active in the studied potential intervals. Moreover, the concentrations of AA and UA are much higher than that of DA and SER in body fluids. However, in our previous studies, we have achieved the discrimination of oxidation potentials of AA, DA and UA by the ETPG electrode [12-13]. From these substances, the oxidation potential of UA was much close to that of SER at ETPG electrode. Therefore, the effect of the measurement medium $\mathrm{pH}$ on the oxidation of SER was investigated in the absence and presence of UA. Firstly, the $\mathrm{pH}$ value of 7.4 was examined 
because the aim of the study was the determination of SER in blood serum, which has the $\mathrm{pH}$ value of 7.4. The ASDPV of 5.0 $\mu \mathrm{M}$ SER is presented in Figure 2a-A. As can be seen, an oxidation peak was observed at $+0.24 \mathrm{~V}$ indicating the oxidation of SER at ETPG electrode. When the ASDPV measurement was repeated in the presence of $5.0 \mu \mathrm{M}$ UA at the same conditions, as expected, the oxidation of SER and UA took place almost same potential value as in Figure 2a-B. This situation indicates that the determination of SER was not possible in blood serum at this $\mathrm{pH}$ value because of the interference of UA. The effect of $\mathrm{pH}$ on the electrochemical oxidation of SER and UA was continued at different $\mathrm{pH}$ values in the range of 7.4 and 1.6. Figure $2 \mathrm{~b}$ showed that oxidation potential values of both molecules shifted towards more anodic potential values by decreasing the $\mathrm{pH}$ value. When the $\mathrm{pH}$ reached to 1.6, three well-defined oxidation peaks were observed at $+0.48 \mathrm{~V},+0.59 \mathrm{~V}$ and $+0.69 \mathrm{~V}$ (vs SCE) in Figure $2 \mathrm{~b}-\mathrm{B}$. Two of them $(+0.48 \mathrm{~V}$ and $+0.69 \mathrm{~V})$ belong to the oxidation of SER as shown in Figure $2 \mathrm{~b}-\mathrm{A}$ and the other peak $(+0.59 \mathrm{~V})$ corresponds to the oxidation of UA. As seen, there is a $0.110 \mathrm{~V}$ difference between the oxidation potential of SER and the oxidation potential of UA. This potential difference is suitable to determine SER in blood serum, which contains high concentrations of UA with high accuracy and sensitivity.
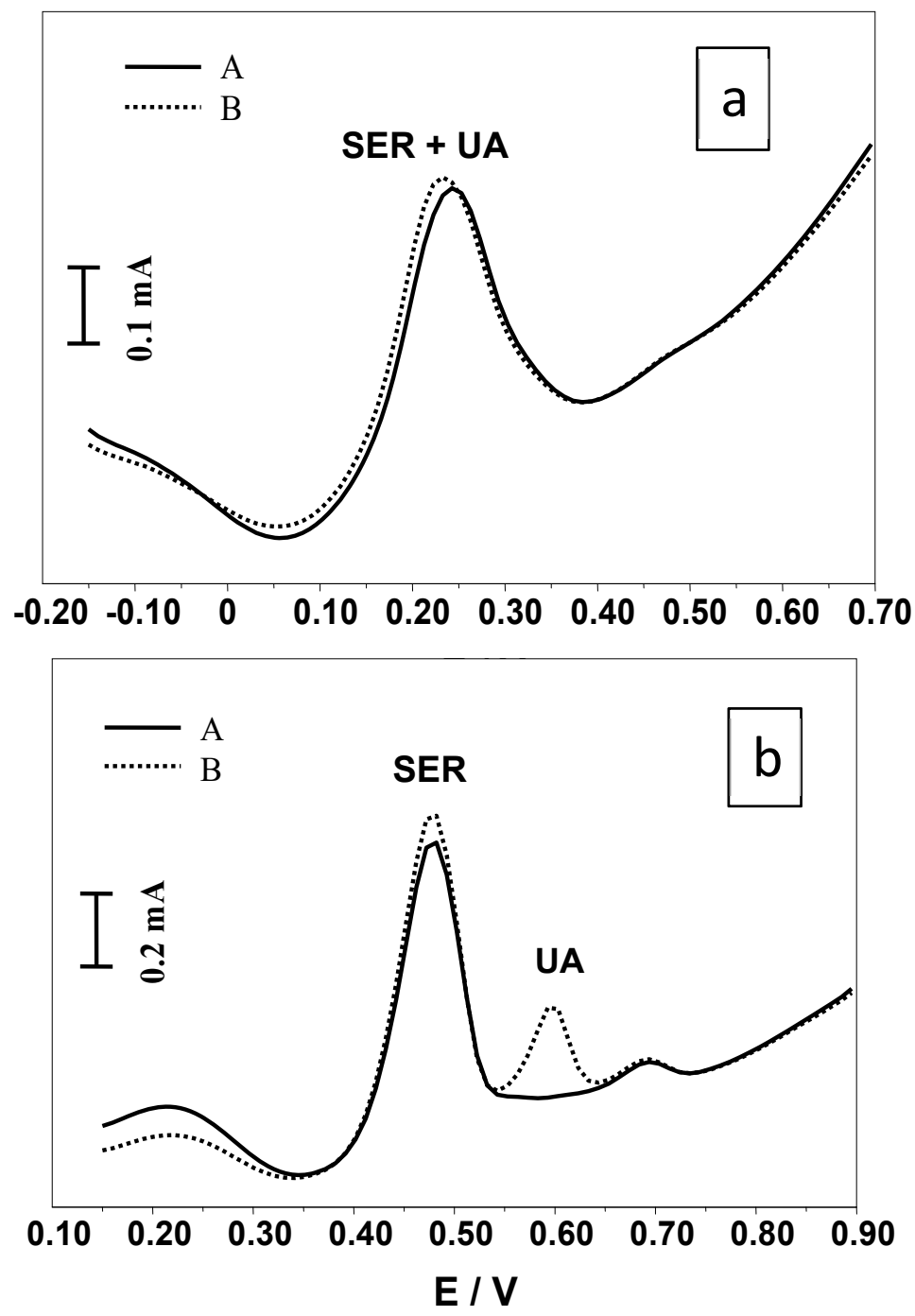

Figure 2. The ASDPVs of 5.0 $\mu \mathrm{M}$ SER in the absence (A) and presence of 5.0 $\mu \mathrm{M}$ UA (B) obtained by ETPG at different $\mathrm{pH}$ values of 7.4 (a) and 1.6 (b). Before the measurement, the ETPG electrode was immersed into measurement solution for $2 \mathrm{~min}$ at stirring conditions ( $250 \mathrm{rpm}$ ) 
The different behaviors of SER and UA at different $\mathrm{pH}$ values can be explained by the $\mathrm{pH}$ dependence of oxidation mechanisms of SER and UA. When the oxidation potential values were plotted against $\mathrm{pH}$, a linear relationship was observed for both molecules between the oxidation potentials and $\mathrm{pH}$. The slopes of the corresponding lines of SER and UA were $-43.0 \mathrm{mV} / \mathrm{pH}$ and $-62.0 \mathrm{mV} / \mathrm{pH}$. The obtained slopes indicate while only one proton taking place in the oxidation process of SER [9,10], two protons take place in the oxidation mechanism of UA [13]. Based on these results and the previous reports $[10,13]$, the corresponding oxidation mechanisms can be shown as in Figure 3. This difference in the oxidation mechanisms of SER (Figure 3a) and UA (Figure 3b) allows the determination of SER in the presence of UA in blood serum samples.

a)

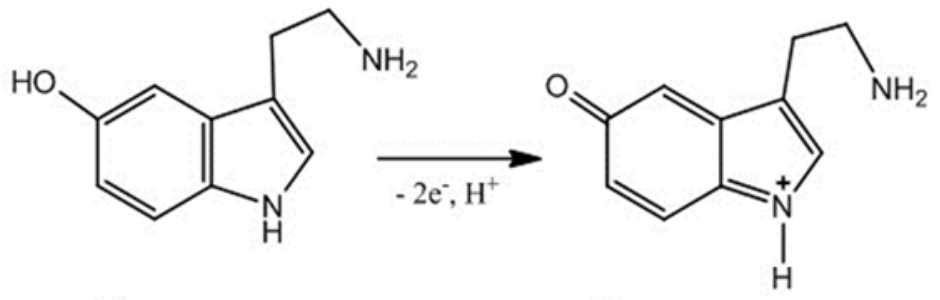

b)

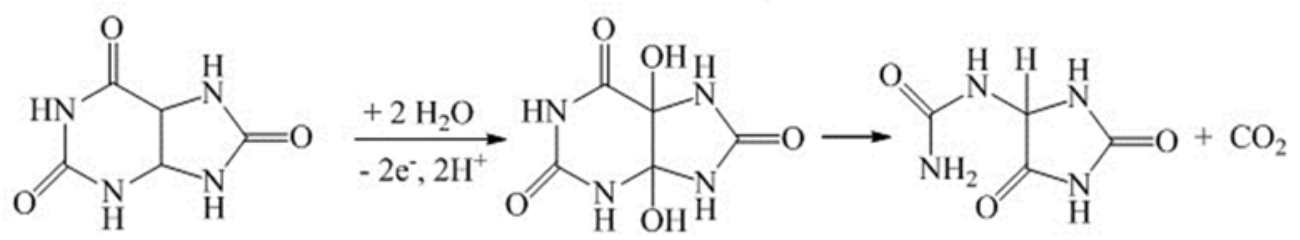

Figure 3. The oxidation mechanisms of SER (a) and UA (b)

The effect of $\mathrm{pH}$ on the oxidation peak current values of SER in the absence of UA was also investigated. When the $\mathrm{pH}$ value of measurement medium was changed from acidic to basic values, the oxidation peak currents of SER were decreased. According to the results, the highest oxidation current values were observed at $\mathrm{pH}$ value of 1.6 and it was used in the rest of the study. On the other hand, the optimal $\mathrm{pH}$ value for the oxidation of SER was reported as 8.06 [6], 7.4 [8] and 7.5 [9]. These results indicated that SER showed different adsorption and oxidation characteristics at the ETPG electrode according to different modified electrodes.

\subsection{The Effect of Accumulation Time}

The magnitude of the oxidation peak currents of SER depends on the surface concentration of SER on the ETPG surface. The surface concentration is directly proportional with the contact time of ETPG in SER solution. As a result, to examine the effect of accumulation time, the ETPG electrodes were immersed into the mixture of $0.1 \mathrm{M} \mathrm{H}_{3} \mathrm{PO}_{4}$ and $0.02 \mathrm{M} \mathrm{LiClO}_{4}$ electrolyte solution (pH 1.6) containing $5.0 \mu \mathrm{M}$ SER for different time periods at stirring conditions $(250 \mathrm{rpm})$. Then the DPV measurements were performed in the same solution. Figure 4 showed that oxidation peak current values of SER were found to increase with increasing accumulation time up to $180 \mathrm{~s}$. When the accumulation time was higher than $180 \mathrm{~s}$, the oxidation current reached a steady-state value. It can be concluded that the optimum accumulation time under these conditions was $180 \mathrm{~s}$. 


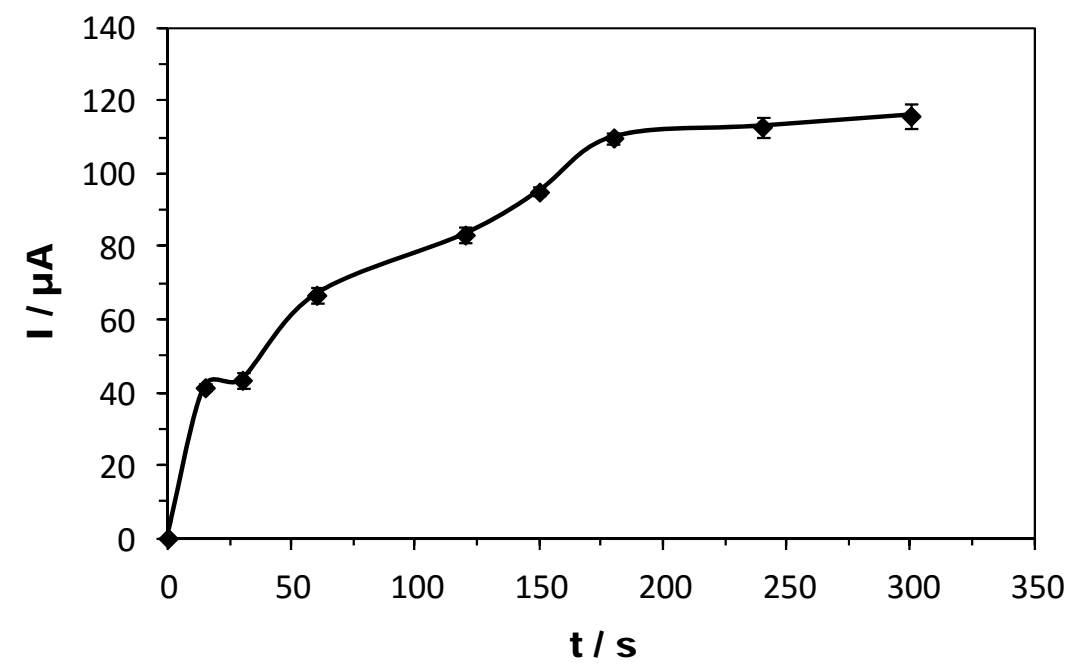

Figure 4. The effect of accumulation time on the oxidation peak currents of SER. Before the measurement, the ETPG electrode was immersed into the mixture of $0.1 \mathrm{M} \mathrm{H}_{3} \mathrm{PO}_{4}$ and 0.02 $\mathrm{M} \mathrm{LiClO}_{4}$ solution (pH 1.6) containing 5.0 $\mu \mathrm{M}$ SER for different time periods at stirring conditions $(250 \mathrm{rpm})$

\subsection{Interference Studies}

The performance of the ETPG electrode mainly depends on the discrimination of SER signals from the interfering species, which is commonly present in the real samples. To test this property of the ETPG electrode, the determination of SER was performed in solutions, which contain some of the possible interfering substances. The selectivity limit was defined as the concentration ratio of interfering / SER causing less than $\pm 5.0 \%$ relative error. Blood serum contains different electroactive compounds such as dopamine (DA), ascorbic acid (AA) and uric acid (UA). These three molecules are oxidized at the potential values, which is close to that of SER. Therefore, their interference effects on the response of SER were firstly investigated. In this frame, the ASDPV studies of SER $(1.0 \mu \mathrm{M})$ were conducted in the absence and presence of the selected interfering molecules. The presence of five-fold DA, hundred-fold UA and thousand-fold AA concentrations did not affect the peak current intensity of SER.

The glucose and urea concentrations, which are two thousand times higher than SER concentration, produced negligible changes in the oxidation peak current intensity of SER.

The change in the magnitude of the oxidation peak current of $1.0 \mu \mathrm{M}$ SER was negligible in the presence of tartaric acid $(250 \mu \mathrm{M})$, citric acid $(250 \mu \mathrm{M})$, sodium chloride $(1000 \mu \mathrm{M})$ and potassium chloride $(1000 \mu \mathrm{M})$.

To test the effects of blood serum proteins, the oxidation of SER was examined in the absence and presence of bovine serum albumin, which constitutes about $60 \%$ of the total protein in blood serum [18]. The peak current intensity of SER decreased almost two-fold in the presence of $1.25 \mathrm{mg} \mathrm{mL}^{-1} \mathrm{BSA}$ concentration in the measurement medium. No further change was observed in the oxidation peak current of SER by increasing the BSA concentration up to $5.0 \mathrm{mg} \mathrm{mL}^{-1}$, which corresponds to BSA concentration in ten-fold diluted blood serum. It is important to note that the presence of BSA decreases the sensitivity of ETPG electrode but it does not prevent the determination of SER completely in blood serum samples. 
Özcan / Anadolu Univ. J. of Sci. and Technology - A - Appl. Sci. and Eng. 17 (3) - 2016

\subsection{Calibration Graph and Detection Limit}

The relationship between the oxidation peak currents and the SER concentration was determined by performing a series of ASDPVs. The oxidation current value was found to increase with increasing SER concentration. It was observed that there were two linear regions in the calibration graph of SER. The first linear region was observed for the concentration values between $0.005 \mu \mathrm{M}$ and $1.0 \mu \mathrm{M}$ and the equation of this line was $\mathrm{I}(\mu \mathrm{A})=44.46 \mathrm{C}(\mu \mathrm{M})\left(\mathrm{R}^{2}: 0.9947\right)$. The second linear relationship was seen over the concentration range of about $2.5 \mu \mathrm{M}$ and $10.0 \mu \mathrm{M}$ with an equation of $\mathrm{I}(\mu \mathrm{A})=10.31 \mathrm{C}(\mu \mathrm{M})$ $+58.05\left(\mathrm{R}^{2}: 0.9942\right)$. A comparison between the obtained slopes of lines showed that the first linear region was more sensitive than the second region. The detection limit value was determined as $1.0 \mathrm{nM}$ according to $3 \sigma$ rule.

Table 1 summarizes the linear range and detection limit values of the presented work and previously published studies. The obtained linear dynamic range for ETPG electrode was comparable with that of the literature values. Moreover, the lowest detection limit was obtained by the proposed method.

The fabrication reproducibility of ETPG electrodes were tested by preparing ETPG and measuring 1.0 $\mu \mathrm{M}$ SER under the same conditions. An RSD value of $2.8 \%$ was obtained after ten measurements. The results reveal that the ETPG electrode has good operational reproducibility.

Table 1. A comparison of the linear range and detection limit values of ETPG with the literature studies

\begin{tabular}{llll}
\hline Electrode & Linear range $(\boldsymbol{\mu M})$ & Detection limit $(\boldsymbol{\mu M})$ & Reference \\
\hline${ }^{\mathrm{a}} \mathrm{GC}-\mathrm{C} 4 \mathrm{R}$ & $0.1-10$ & 0.03 & 5 \\
${ }^{\mathrm{b}} \mathrm{GC}-\mathrm{E}$ GOP & $0.1-300$ & 0.0049 & 6 \\
${ }^{\mathrm{c}} \mathrm{GC}-\mathrm{CNFs}$ & $1.0-10$ & 0.25 & 7 \\
${ }^{\mathrm{d}} \mathrm{Nafion} / \mathrm{Co}(\mathrm{OH})_{2}-$ & $0.05-75$ & 0.023 & 8 \\
MWCNTs/CILE & $0.1-100$ & 0.03 & 9 \\
${ }^{\mathrm{e}} \mathrm{PMEPGE}$ & $0.01-1.0$ & 0.0025 & 19 \\
${ }^{\mathrm{f} P} 3 \mathrm{CA}-\mathrm{PGE}$ & $0.005-1.0$ & 0.001 & This study \\
${ }^{\mathrm{g}} \mathrm{ETPG}$ & & & \\
\hline
\end{tabular}

${ }^{\mathrm{a}}$ Glassy carbon-C-undecylcalix[4]resorcinarene.

${ }^{\mathrm{b}}$ Glassy carbon electrochemically reduced graphene oxide-porphyrin.

${ }^{\mathrm{c}}$ Glassy carbon-carbon nanofibers.

${ }^{\mathrm{d}} \mathrm{Nafion} / \mathrm{Co}(\mathrm{OH})_{2}$-multiwalled carbon nanotubes modified carbon ionic liquid

electrode.

ePolymelamine modified edge plane pyrolytic graphite.

fPoly(pyrrole-3-carboxylic acid) modified pencil graphite electrode.

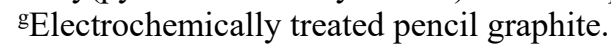

\subsection{Analytical Applications}

The analytical application of the proposed method was tested in blood serum samples. Before the voltammetric measurements, blood serum samples were diluted forty-fold by the mixture of $0.1 \mathrm{M}$ $\mathrm{H}_{3} \mathrm{PO}_{4}$ and $0.02 \mathrm{M} \mathrm{LiClO}_{4}$ solution (pH 1.6). The ETPG electrode was immersed in this solution for 3 min. After that, the DPV measurement was performed in the same solution. The concentration of SER in the measurement medium was gradually increased by the addition of standard SER solution. The obtained ASDPVs for one of the samples are shown in Figure 5. The calibration curve equations of two different blood serum samples were calculated as $I(\mu \mathrm{A})=28.99 \mathrm{C}(\mu \mathrm{M})+0.284\left(\mathrm{R}^{2}: 0.9997\right)$ and $\mathrm{I}$ $(\mu \mathrm{A})=27.74 \mathrm{C}(\mu \mathrm{M})+0.109\left(\mathrm{R}^{2}: 0.999\right)$. The SER contents of these samples were determined as 0.392 $\mu \mathrm{M}$ and $0.312 \mu \mathrm{M}$, respectively. The same measurement was repeated by using untreated PG instead of ETPG, but the oxidation of SER was not obtained. 


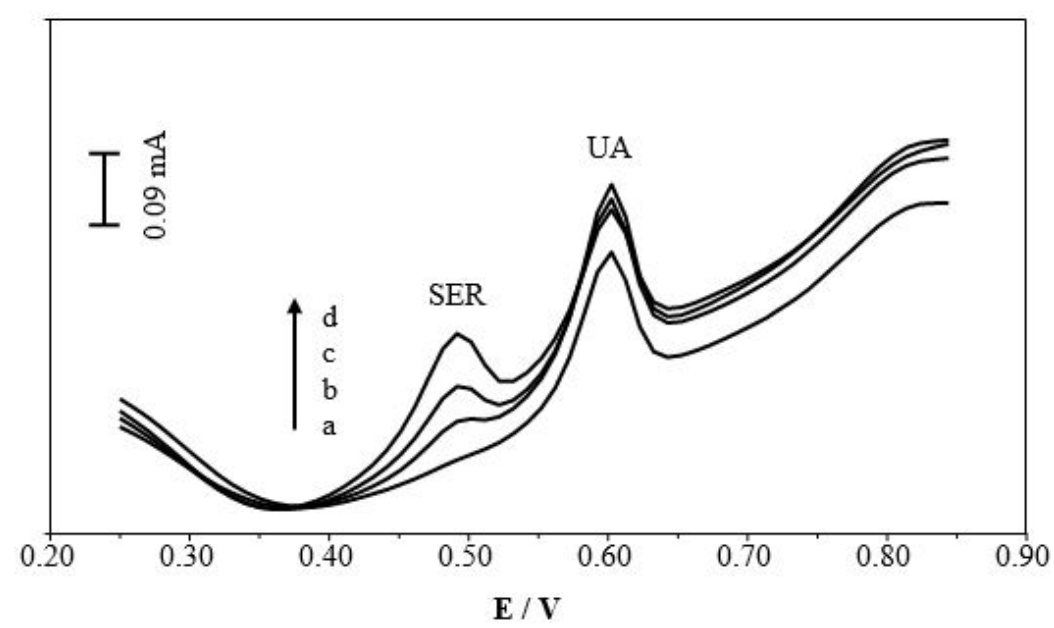

Figure 5. The ASDPVs of ETPG electrode obtained in forty-folded diluted blood serum with the mixture of $0.1 \mathrm{M} \mathrm{H}_{3} \mathrm{PO}_{4}$ and $0.02 \mathrm{M} \mathrm{LiClO}_{4}$ solution $(\mathrm{pH} 1.6)$ in the presence of $0.0 \mu \mathrm{M}$ (a), $0.10 \mu \mathrm{M}(\mathrm{b}), 0.25 \mu \mathrm{M}(\mathrm{c})$ and $0.50 \mu \mathrm{M}$ (d) SER. Before the measurement, the ETPG electrode was immersed into measurement solution for $3 \mathrm{~min}$ at stirring conditions $(250 \mathrm{rpm})$

To verify the reliability of the proposed method, a parallel analysis was also performed with a previously published HPLC analysis method [16]. For this purpose, three human blood serum samples were taken from three volunteers (two male, one female). Before the HPLC measurements, blood serum proteins had to be removed from the analysis medium. Therefore, the blood serum proteins were precipitated by $30 \% \mathrm{HClO}_{4}$ and separated by centrifugation. The $20 \mu \mathrm{L}$ supernatant was injected onto the column and HPLC analysis was performed. The presence of SER in the samples was verified by the increase of the peak observed at the retention time value of $8.13 \mathrm{~min}$ in the related chromatograms as a result of successive addition of standard SER solution. The SER content of the blood serum samples were given in Table 2 .

Table 2. The amount of SER in blood serum samples determined by HPLC and proposed method

\begin{tabular}{|c|c|c|}
\hline \multirow{2}{*}{ Sample ID } & \multicolumn{2}{|c|}{ [SER] / $\boldsymbol{M M}$} \\
\cline { 2 - 3 } & HPLC method & Proposed method \\
\hline A & $0.58 \pm 0.05$ & $0.57 \pm 0.06$ \\
\hline B & $0.48 \pm 0.06$ & $0.43 \pm 0.05$ \\
\hline C & $0.31 \pm 0.04$ & $0.29 \pm 0.03$ \\
\hline
\end{tabular}

The same protein-free samples were also analyzed by the proposed method to maintain the comparability of both methods. The $1000 \mu \mathrm{L}$ protein-free supernatant was transferred into the electrochemical cell and diluted ten-fold by the mixture of $0.1 \mathrm{M} \mathrm{H}_{3} \mathrm{PO}_{4}$ and $0.02 \mathrm{M} \mathrm{LiClO}_{4}$ solution (pH 1.6). The ETPG electrode was immersed in this solution for $3 \mathrm{~min}$. After that, the DPV measurement was performed in the same solution. The concentration of SER in the measurement medium was gradually increased by the addition of standard SER solution. As can be seen from Figure 6, two oxidation peaks were observed at the peak potential of $+0.516 \mathrm{~V}$ and $+0.632 \mathrm{~V}$ (vs. SCE) in blood serum. The peak current observed at $+0.516 \mathrm{~V}$ increased by the successive addition of standard SER solutions. This reveals that this peak $(+0.516 \mathrm{~V})$ arises from the oxidation of SER. On the other hand, the peak observed at $+0.632 \mathrm{~V}$ is arising from the oxidation of UA found in blood serum. Moreover, the oxidation peak potentials of SER and UA shifted towards anodic direction in the case of protein-free blood serum samples according to 
their standard solutions in $0.1 \mathrm{M} \mathrm{H}_{3} \mathrm{PO}_{4}\left(\mathrm{pH}\right.$ 1.6) solution containing $0.02 \mathrm{M} \mathrm{LiClO}_{4}$. This situation may arise from the $\mathrm{pH}$ change of the analysis medium in the case of protein-free blood serum samples. The use of $30 \% \mathrm{HClO}_{4}$ solution to precipitate the blood serum proteins decreases the $\mathrm{pH}$ of the analysis medium. This led to the shift of oxidation potentials towards anodic direction. The analysis results of three samples are given in Table 2. No DA peak was observed at these conditions. These results show that the determination of SER in blood serum samples can be successfully performed by ETPG electrode.

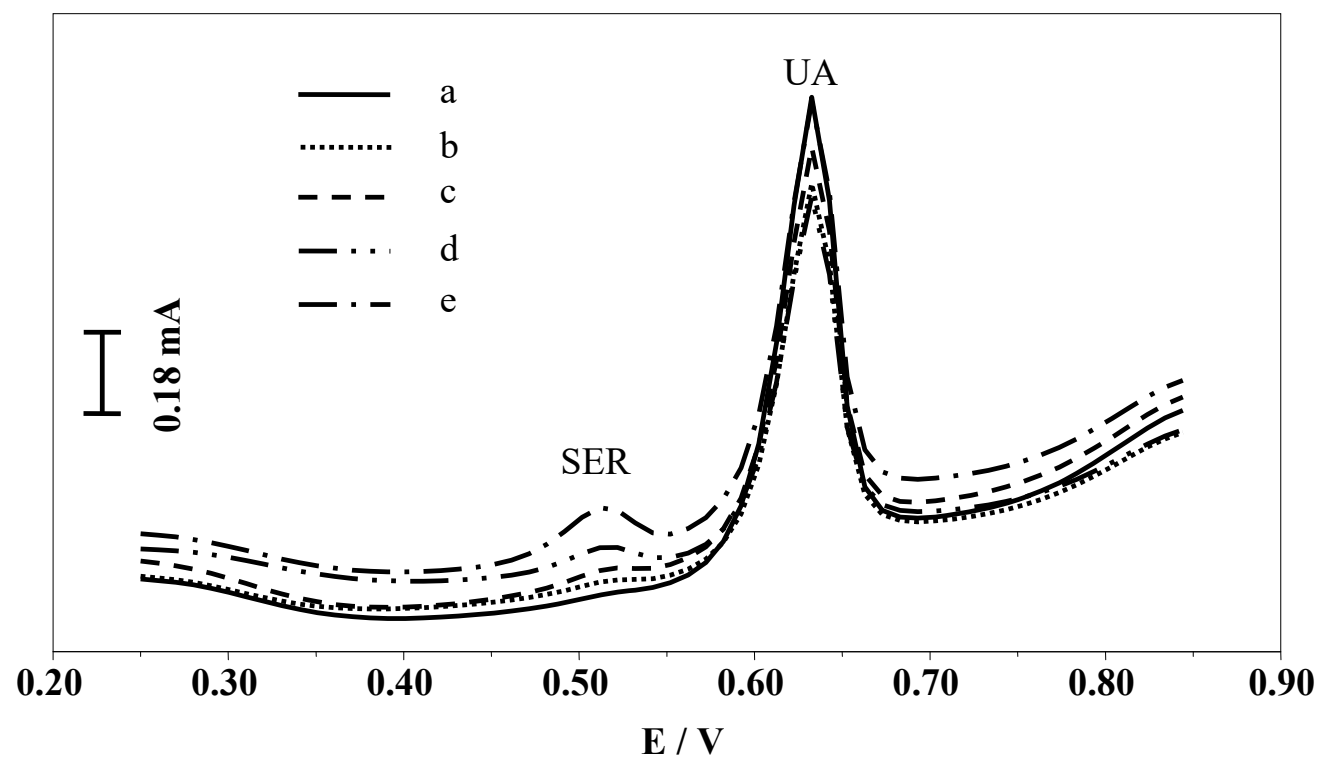

Figure 6. The ASDPVs of ETPG electrode obtained in ten-folded diluted protein free blood serum (proteins were precipitated with $30 \% \mathrm{HClO}_{4}$ ) with the mixture of $0.1 \mathrm{M} \mathrm{H}_{3} \mathrm{PO}_{4}$ and $0.02 \mathrm{M}$ $\mathrm{LiClO}_{4}$ solution ( $\mathrm{pH}$ 1.6) in the presence of $0.0 \mu \mathrm{M}$ (a), $0.05 \mu \mathrm{M}$ (b), $0.10 \mu \mathrm{M}$ (c), $0.25 \mu \mathrm{M}$ (d) and $0.50 \mu \mathrm{M}$ (e) SER. Before the measurement, the ETPG electrode was immersed into measurement solution for 3 min at stirring conditions $(250 \mathrm{rpm})$

As can be seen from Table 2, the obtained SER concentrations for both methods are much closer to each other. Moreover, these values are in accordance with the normal range $(0.573 \mu \mathrm{M}-1.606 \mu \mathrm{M})$ of SER in blood serum.

A statistical test (Kruskal Wallis Test) was applied to the data obtained both HPLC and proposed DPV methods to evaluate the presence of a systematic error. Kruskal Wallis Test was applied to the related data sets using a statistical packed program (SSPS Statistics Version 22). The obtained results are given in Table 3. The obtained value of 0.563 for chi-square (Table 3 ) was smaller than the theoretical chisquare value (3.841 for $95 \%$ confidence level and 1 degree of freedom). This indicates that there is no significant difference between the results of both HPLC and DPV methods indicating no systematic error in the proposed method.

Table 3. The results of Kruskal Wallis Test applied to data obtained during the determination of SER in blood serum by both HPLC and proposed DPV methods

\begin{tabular}{|l|l|}
\hline Kruskal Wallis Test* \\
\hline Chi-Square & 0.563 \\
\hline Degree of freedom & 1 \\
\hline Asymptotic Significance & 0.453 \\
\hline *The values obtained by a packed program (SPSS statistics Version 22) \\
\hline
\end{tabular}


Özcan / Anadolu Univ. J. of Sci. and Technology - A - Appl. Sci. and Eng. 17 (3) - 2016

\section{CONCLUSIONS}

In conclusion, the ETPG electrode showed good electrocatalytic activity towards the oxidation of SER, as well as its high SER selectivity and sensitivity. The ETPG electrode achieved the discrimination of SER and UA, which was not observed in the case of bare PG electrode. The results indicated that $\mathrm{pH}$ of the measurement medium was very important for the selective determination of SER in blood serum samples to eliminate the interference of UA. The proposed method has been successfully applied to the direct determination of SER in human blood serum samples in the presence of high concentrations of UA. The ETPG electrodes are very promising in the electroanalytical applications due to their low cost, easy preparation procedure and disposable character. The studies on the increment of SER selectivity and sensitivity of ETPG electrode are under study in our laboratory.

\section{REFERENCES}

[1] Ramon-Marquez T, Medina-Castillo AL., Fernandez-Gutierrez A, Fernandez-Sanchez JF. A novel optical biosensor for direct and selective determination of serotonin in serum by Solid Surface-Room Temperature Phosphorescence Biosens. Bioelectron., 2016; 82:217-23.

[2] Wu K, Fei J, Hu S. Simultaneous determination of dopamine and serotonin on a glassy carbon electrode coated with a film of carbon nanotubes Anal. Biochem., 2003; 318:100-6.

[3] Gregersen K, Froyland L, Berstad A, Araujo P. Direct determination of serotonin in gut lavage fluid by liquid chromatographic ion trap tandem mass spectrometry Talanta, 2008; 75:466-72.

[4] Anderson GM, Hall LM, Yang JX, Cohen DJ. Platelet dense granule release reaction monitored by high-performance liquid chromatography-fluorometric determination of endogenous serotonin Anal. Biochem., 1992; 206: 64-7.

[5] Wang F, Wu Y, Lu K, Ye B. A simple but highly sensitive and selective calixarene-based voltammetric sensor for serotonin Electrochim. Acta, 2013; 87:756-62.

[6] Han HS, Lee HK, You JM, Jeong H, Jeon S. Electrochemical biosensor for simultaneous determination of dopamine and serotonin based on electrochemically reduced GO-porphyrin Sens. Actuat. B: Chem., 2014; 190:886-95.

[7] Rand E, Periyakaruppan A, Tanaka Z, zhang DA, Marsh MP, Andrews RJ, Lee KH, Meyyappan M, Koehne JE. A carbon nanofiber based biosensor for simultaneous detection of dopamine and serotonin in the presence of ascorbic acid Biosens. Bioelectron., 2013; 42:434-8.

[8] Kim SK, Kim D, Jeon S. Electrochemical determination of serotonin on glassy carbon electrode modified with various graphene nanomaterials Sens. Actuat. B: Chem., 2012; 174:285-91.

[9] Babaei A, Taheri AR, Aminikhah M. Nanomolar simultaneous determination of levodopa and serotonin at a novel carbon ionic liquid electrode modified with $\mathrm{Co}(\mathrm{OH})_{2}$ nanoparticles and multi-walled carbon nanotubes Electrochim. Acta, 2013; 90:317-25.

[10] Gupta P, Goyal RN. Polymelamine modified edge plane pyrolytic graphite sensor for the electrochemical assay of serotonin Talanta, 2014; 120:17-22.

[11] Thiagarajan S, Tsai TH, Chen SM. Easy modification of glassy carbon electrode for simultaneous determination of ascorbic acid, dopamine and uric acid Biosens. Bioelectron., 2009; 24:2712-5. 
[12] Özcan A, Şahin Y. Selective and sensitive voltammetric determination of dopamine in blood by electrochemically treated pencil graphite electrodes Electroanalysis., 2009; 19:267-75.

[13] Özcan A, Şahin Y. Preparation of selective and sensitive electrochemically treated pencil graphite electrodes for the determination of uric acid in urine and blood serum Biosens. Bioelectron., 2010; 25:2497-502.

[14] Özcan A, Şahin Y. A novel approach for the determination of paracetamol based on the reduction of N-acetyl-p-benzoquinoneimine formed on the electrochemically treated pencil graphite electrode Anal. Chim. Acta., 2011; 685:9-14.

[15] Özcan A, Şahin Y. A novel approach for the selective determination of tryptophan in blood serum in the presence of tyrosine based on the electrochemical reduction of oxidation product of tryptophan formed in situ on graphite electrode Biosens. Bioelectron., 2012; 31:26-31.

[16] Tagari PC, Boullin DJ, Davies CL. Simplified determination of serotonin in plasma by liquid chromatography with electrochemical detection Clin. Chem., 1984; 30:131-5.

[17] Marken F, Eklund JC, Compton RG. Voltammetry in the presence of ultrasound - can ultrasound modify heterogeneous electron-transfer kinetics J. Electroanal. Chem., 1995; 395:335-9.

[18] Shen Y, Jacobs JM, Camp DG, Fang R, Moore RJ, Smith RD, Xiao W, Davis RW, Tompkins RG. Ultra-high-efficiency strong cation exchange LC/RPLC/MS/MS for high dynamic range characterization of the human plasma proteome Anal. Chem., 2004; 76:1134-44.

[19] Özcan A, İlkbaş S. Poly(pyrrole-3-carboxylic acid)-modified pencil graphite electrode for the determination of serotonin in biological samples by adsorptive stripping voltammetry Sens. Actuat. B: Chem., 2015; 215:518-24. 